\title{
Malnutrition Aigüe Sévère et caractéristiques socio-économiques des mères en Commune VI du district de Bamako au Mali
}

\section{Severe Acute Malnutrition and socio-economic characteristics of mothers in Commune VI of the district of Bamako in Mali}

\author{
Diallo $\mathrm{O}^{1^{*}}$, Coulibaly $\mathrm{CA}^{2}$, Diawara $\mathrm{F}^{3,4}$, Fomba $\mathrm{S}^{1}$, Sangho $\mathrm{F}^{4}$, Sogoba $\mathrm{B}^{5}$, Koné $\mathrm{S}^{6}$, Touré $\mathrm{F}^{7}$, Traoré $\mathrm{D}^{4}$, Coulibaly $\mathrm{C}^{4}$, Sangho $\mathrm{O}^{4}$, \\ Sanogo $\mathrm{O}^{2}$, Sangho $\mathrm{A}^{8}, \mathrm{Ag}$ lknane $\mathrm{A}^{3,4}$, Sangho $\mathrm{H}^{2}$
}

1. Population Service International Mali (PSI Mali), Bamako, Mali

2. Faculté de Médecine et d'Odonto-Stomatogie (FMOS), Bamako, Mali

3. Institut National de Santé Publique (INSP), Bamako, Mali

4. Département d'Enseignement et de Recherche des Sciences Biologiques et Médicales, Faculté de Pharmacie (FAPH) /USTTB, Bamako, Mali

5. Centre Hospitalo-Universitaire Gabriel Toure (CHU GT), Bamako, Mali

6. Hôpital Garnison de Kati, Bamako, Mali

7. Programme National de lutte contre le Paludisme (PNLP), Bamako, Mali

8. Département d'Enseignement et de Recherche des Sciences Pharmaceutiques, FAPH/USTTB, Mali

*Auteur principal: Dr Oumar DIALLO, MD, MPH,

CoordínateurRégional MTN/Paludisme PSI Mali. Email :

oumar812@yahoo.fr, Tél:22375246764/69328125

\section{Résumé :}

Introduction : Selon le Programme alimentaire mondial en 2015, il y'avait 795 millions de personnes qui souffraient de la faim dans le monde, dont la grande majorité dans les pays en développement où $13,5 \%$ de la population est sous-alimentée. La malnutrition provoque la mort de 3,1 millions d'enfants de moins de 5 ans chaque année dans le monde. Au Mali, selon la cinquième édition de l'enquête démographique et de santé du Mali, la malnutrition aigüe affecte $13 \%$ des enfants de moins de 5 ans dont $5 \%$ sous la forme sévère. Objectif : Evaluer les facteurs prédisposant à la malnutrition aigüe sévère chez les enfants de 6 à 59 mois à l'Unité de Récupération Nutritionnelle en Intensive du Centre de Santé de Référence de la commune VI de Bamako. Matériel et méthodes : Il a été mené une enquête auprès des enfants malnutris aigus sévères hospitalisés à l'URENI et leurs mères du 06 octobre au 30 novembre 2015. Résultats : Le quartier de Senou a enregistré le plus grands nombre d'enfants malnutris aigus sévères $(20 \%)$. La majorité des mères avait le niveau primaire ou n'avait aucun niveau, avec respectivement $55 \%$ et $32 \%$. Les mères d'enfants dans la plupart des cas étaient des ménagères $(60 \%)$ et n'avaient pas d'activité génératrice de revenu $(63 \%)$. Conclusion : Dans cette étude, les mères des enfants dans la plupart des cas étaient des ménagères et n'avaient pas d'activité génératrice de revenu.

Mots Clés : malnutrition aigüe sévère, enfants de 6-59 mois, mères, URENI, Bamako, Mali.

\footnotetext{
Abstract:

Introduction: According to the World Food Program in 2015, there were 795 million hungry people in the world, the vast majority of them in developing countries where
}

$13.5 \%$ of the population is undernourished. Malnutrition kills 3.1 million children under the age of 5 each year worldwide. In Mali, according to the fifth edition of the Mali Demographic and Health Survey, Acute malnutrition affects $13 \%$ of children under the age of $5,5 \%$ of whom are in severe form. Objective: To assess the factors predisposing to severe acute malnutrition in children aged 6 to 59 months at the Intensive Nutritional Recovery Unit of the Reference Health Center of the commune VI of Bamako. Material and methods: A survey was carried out among severe acute malnourished children hospitalized at URENI and their mothers from 06 October to 30 November 2015. Results: The Senou district recorded the largest number of severe acute malnourished children $(20 \%)$. The majority of mothers had primary education or had no education at $55 \%$ and $32 \%$ respectively. Mothers of children in most cases were housewives $(60 \%)$ and had no income-generating activity (63\%) Conclusion: In this study mothers of children in most cases were housewives and had no incomegenerating activity.

Key words: severe acute malnutrition, children aged 6-59 months, mothers, a recovery unit and intensive nutritional education, Bamako, Mali.

\section{Introduction}

Selon l'Organisation mondiale de la santé (OMS) : «la malnutrition est un état pathologique résultant de l'insuffisance ou des excès relatifs ou absolus d'un ou de plusieurs nutriments essentiels, que cet état se manifeste cliniquement ou qu'il ne soit décelable que par les analyses biologiques, anthropométriques ou physiologiques » (1).

La malnutrition est causée par une combinaison de facteurs tels que le revenu faible, l'analphabétisme, l'environnement insalubre, les services de santé insuffisants, les habitudes alimentaires inadéquates et la faible productivité agricole. Tous ces facteurs s'influencent réciproquement, mais de manière spécifique (2).

La malnutrition n'est pas seulement un problème de santé. Elle fait partie d'un cercle vicieux bien plus large : l'ampleur des dysfonctionnements de l'état nutritionnel dus à la malnutrition peut affecter le développement physique et mental de l'enfant, qui déterminent plus tard sa potentialité d'insertion dans la vie socio-économique (3).

Elle a donc des répercussions économiques et sanitaires très importantes tant au niveau communautaire qu'à l'échelle de la nation, sans compter que les femmes ayant souffert de malnutrition donneront naissance à des 
enfants de faible poids, le cycle se reproduisant ainsi sans fin (3).

En 2015, selon le Programme alimentaire mondial (PAM), 795 millions de personnes souffraient de la faim dans le monde, soit 1 personne sur 9. La grande majorité des personnes souffrant de la faim vivaient dans des pays en développement où $13,5 \%$ de la population est sousalimentée. L'Asie comprend deux tiers des personnes sous-alimentées dans le monde. L'Afrique subsaharienne est la région avec la plus forte prévalence (pourcentage de la population) de la faim (4). Une personne sur quatre y est sous-alimentée. La malnutrition provoque la mort de 3,1 millions d'enfants de moins de 5 ans chaque année, soit près de la moitié $(45 \%)$ des causes de décès. Un enfant sur six, soit 100 millions d'enfants, souffre d'insuffisance pondérale dans les pays en voie de développement. Un enfant sur quatre souffre de retards de croissance. Dans les pays en voie de développement, ce chiffre peut atteindre un enfant sur trois (4).

$\mathrm{Au}$ Mali, selon la cinquième édition l'Enquête démographique et de santé du Mali (EDSM V), $38 \%$ des enfants de moins de 5 ans présentent une malnutrition chronique dont $19 \%$ sous la forme sévère. La malnutrition aigüe affecte $13 \%$ des enfants de moins de 5 ans dont $5 \%$ sous la forme sévère. Plus d'un quart des enfants de moins de 5 ans $(26 \%)$ présentent une insuffisance pondérale. Dans $9 \%$ des cas, il s'agit d'une insuffisance pondérale sévère (5). Cette situation peu reluisante, malgré les efforts de l'état et ses partenaires, nous a incité à mener une étude afin de déterminer les facteurs prédisposant à la malnutrition aigüe sévère chez les enfants de 6 à 59 mois hospitalisés à l'URENI (Unité de récupération nutritionnelle en intensive) du Centre de Santé de Référence de la commune VI du district de Bamako entre octobre-novembre 2015.

\section{Matériel et méthodes \\ Cadre et type d'étude :}

II s'agissait d'une étude observationnelle descriptive transversale qui s'est déroulée à l'URENI du Centre de santé de Référence de la commune VI de Bamako du 06 octobre au 30 novembre 2015.

\section{Population d'étude et échantillon :}

La population d'étude était constituée par les enfants malnutris aigue sévères hospitalisés à l'URENI durant la période de l'étude ainsi que leurs mères. Nous avons procédé à une enquête exhaustive de tous les enfants malnutris aigus sévères hospitalisés à l'URENI durant la période de l'étude ainsi que leurs mères.

\section{Critères d'inclusion :}

Tous les enfants de 6-59 mois malnutris aigus sévères hospitalisés durant la période d'étude, dont les mères ont accepté de participer à l'étude.

\section{Critères de non inclusion :}

Les enfants de moins de 6 mois et de plus de 59 mois malnutris sévères hospitalisés et ceux de 6-59 mois dont les mères ont refusé de participer à l'étude.
Outils de collecte des données :

Nous avons utilisé des questionnaires a plusieurs volets, administrés en mode face à face et des instruments de mesures anthropométriques.

Variables d'étude :

Les variables étudiées étaient les suivantes : âge de l'enfant, périmètrebrachiale, taille, poids, caractéristiques sociodémographiques et économiques de la mère.

Analyse des données :

Toutes les données ont été saisies sur EPI data version 3.1 et analysées sur ENA for SMART pour les données anthropométriques et le reste de l'analyse sur le logiciel SPSS version 21. Nous avons utilisé le chi 2 comme test statistique.

\section{Aspects éthiques :}

Le consentement verbal des mères des enfants a été requis, avant l'inclusion de leurs enfants dans cette étude. Le respect de la confidentialité des données utilisées a été observé.

\section{Résultats}

Les résultats relevant les aspects socioéconomiques et les conditions d'hygiène et d'assainissement de la famille de l'enfant. L'âge moyen des mères était de 24 -34 soit $94 \%$; l'âge minimum 16 ans $3 \%$ et la maximum 43 ans $3 \%$. Le sexe ratio des enfants était de 1,06 en faveur du féminin. Presque toutes les mères étaient mariées, soit 97 $\%$. La plupart des mères d'enfant avait un niveau primaire soit $55 \%$ et $32 \%$ aucun. Les femmes au foyer ont représenté $60 \%$ de l'effectif. (tableau I).

Les mères en général utilisaient des latrines traditionnelles, soit $77 \%$ de l'effectif. La plupart de mères ont déclaré se laver les mains au savon après avoir nettoyé l'enfant, soit $74 \%$ des cas. (tableau II).

La plupart des femmes avait une période inter génésique de 2 ans et plus soit $63 \%$. (tableau III).

Les femmes ne réalisaient pas d'AGR dans $62,9 \%$ des cas.

Un seul Médecin dirigeait l'URENI et est formé sur le protocole national de prise en charge de la Malnutrition. En ce qui concerne les infirmiers, 2 ont reçu une formation et les autres ont appris sur le tas (tableau V).

\section{Discussion}

Limites de l'étude : La population n'est pas trop grande et la période d'étude aussi est courte. En plus, l'étude a concerné seulement l'URENI du CSREF de la Commune VI de Bamako.

L'âge des mères: Dans notre étude, l'âge moyen des mères a été de 24,34 ; l'âge minimum a été de 16 ans et le maximum 43 ans. Ce résultat est proche de ceux obtenus par FALL LO A au Sénégal $(27,4 \%)$ (6) et DIABATE F au CHU Gabriel TOURE (27.6\%) (6).

Niveau d'éducation : Dans cette étude la plupart des mères d'enfant avait un niveau primaire soit $54,3 \%$ et $31,4 \%$ n'avaient aucun niveau d'alphabétisation. FALL LO A au Sénégal en 2011 a trouvé que la majorité des mères $(66,7 \%)$ étaient soit analphabètes ou avaient un faible 
niveau d'instruction (6). DIABATE F au CHU Gabriel TOURE en 2010 a aussi trouvé que $94,2 \%$ des mères n'avaient aucun niveau d'instruction (7). Ce résultat est diffèrent aussi de celui de Bissan. A qui avait trouvé dans le cercle de Nara en 2013 que 71,9\% des mères n'avaient aucune instruction (8). Donc le faible niveau d'instruction semble constituer un facteur influant négativement ce phénomène. En effet, l'analphabétisme, la grande taille du ménage ainsi que les bas revenus conduisent tous à un déséquilibre nutritionnel, voire une malnutrition (6)

Profession des mères : Les femmes au foyer ont représenté $60 \%$ de l'effectif. Ceci est inférieur à celui obtenu par FALL LO A au Sénégal en $2011(77,7)$ (6). Bien qu'elles soient plus disponibles pour s'occuper de leur enfant sur le plan nutritionnel, elles peuvent cependant manquer de ressources pour contribuer à l'amélioration de l'alimentation de leur famille sur le plan qualitatif et quantitatif. L'absence d'occupation professionnelle des mères est certes une opportunité sur le plan disponibilité temporelle pour s'occuper de leur progéniture, mais également un obstacle socioéconomique pour la disponibilité alimentaire. En effet, l'exercice d'une activité professionnelle par la mère accroît les ressources familiales et devrait donc diminuer le risque de malnutrition. Mais cette activité a aussi une incidence négative parce que la mère ne peut pas s'occuper de son jeune enfant pendant la journée (6).

Statut matrimonial : Dans notre étude presque toutes les mères étaient mariées soit $97,1 \%$. Ce résultat est proche de ceux obtenus par FALL LO A au Sénégal en 2011 $(93,3 \%)(6)$ et TIMAK au Burkina $(96,20 \%)(9)$. Le mariage étant un facteur de stabilité sociale, cet aspect pourrait être positif pour la prise en charge nutritionnelle des enfants. La participation de chaque conjoint à accroitre les revenus de la famille devient importante.

Parité : La majorité des femmes était multipare soit $80 \%$. Ce taux est plus élevé que celui obtenu par Fall LO. A au Sénégal en 2011 (65,30\%) (6).

Période inter génésique : Dans notre étude, la plupart des femmes avait une période inter génésique de plus de 2 ans et plus soit $62,9 \%$. Ceci est contraire à celui obtenu par TIMA K au Burkina en 2011, c'est-à-dire 58,23\% avaient une période inter génésique inférieure à 2 ans (22).

Réalisation d'AGR : Les femmes n'avaient pas d'AGR dans $62,9 \%$ des cas. Notre taux est supérieur à celui de l'EDSV 2012-2013 qui avait trouvé que près de la moitié des femmes de 15-49 ans (48\%) ont exercé une activité économique au cours des 12 mois ayant précédé l'enquête.

Type de latrine utilisé : Les mères en général utilisaient des latrines traditionnelles, soit $77,1 \%$ de l'effectif. Selon l'EDSV, seuls $22 \%$ des ménages disposent de toilettes améliorées et non partagées avec une variation de de 41 $\%$ en milieu urbain à $17 \%$ en milieu rural.

Lavage des mains au savon après avoir nettoyé l'enfant : La plupart des mères ont déclaré se laver les mains au savon après avoir nettoyé l'enfant, soit $74,3 \%$ des cas. Ceci est nettement supérieur aux $10 \%$ obtenus par Bissan A dans le cercle de Nara en 2013(8) et également une étude réalisée au Cambodge en 2013 qui a trouvé seulement $6,7 \%(10)$.

\section{Conclusion}

Dans notre étude la plupart des mères était non alphabétisée ou avait le niveau primaire et n'avait pas d'activité génératrice de revenu. Nous pouvons donc déduire que la non alphabétisation, le bas niveau d'instruction, la profession et le manque d'activité génératrice de revenu peuvent constituer des facteurs prédisposant à la malnutrition aiguë sévère.

\section{Références}

1. Weltgesundheits organisation. La prise en charge de la malnutrition sévère : manuel à l'usage des médecins et autres personnels de santé à des postes d'encadrement. Genève ; 2000.63 p.

2. FAO. Nutrition dans les pays en développement. FAO ; 2011.23p

3. GUEYE BY. Suivi nutritionnel des enfants de 06 à 59 mois et ugodesumalnutris des communes de Zangaradougou et Danderesso (cercle de Sikasso). 2012 ; (11P25) :71.

4. PAM. L'Etat de l'insécurité alimentaire dans le monde [Internet]. Rome : FAO ; 2015 [cité 28 janv 2016] p. 66. Report No. : a-i4646f. Disponible sur http://www.fao.org/3/ai4646f.pdf.

5 . Enquête Démographique et de Santé du Mali EDSM-V 2012-2013 [Internet]. [Cité 5 janv 2016]. Disponible sur : https://dhsprogram.com/pubs/pdf/PR33/PR33.pdf

6. LO AF. Facteurs explicatifs de la fréquence de la malnutrition chez les enfants âgés de 0 à 5 ans dans la communauté rurale de NguèneSarr au Sénégal [Internet]. Mémoire Online. 2011 [cité 28 janv 2016]. Disponible sur : http://www.memoireonline.com/12/13/8181/Facteurs-

explicatifs-de-la-frequence-de-lamalnutrition- chez-lesenfants-ges-de-0--5-ans-dans.html

7.Diabaté $\mathrm{F}$ dite Ss. Bilan d'activités de I'URENI des enfants Malnutris Sévères de 0 A 59 mois Hospitalises dans le service de Pédiatrie du CHU Gabriel Toure [Internet]. [Bamako] :FMOS ; 2010 [Cité 28 Janv. 2016]. Disponible Sur Http://Www.Keneya.Net/Fmpos/Theses/2010/Med/Pdf/10 m326.Pdf

8. Bissan A. Sécurité Nutritionnelle dans le Cercle de Nara en2012 [Internet]. [BAMAKO] : FMOS ; 2014 [cité 27 janv 2016]. Disponible sur http://www.keneya.net/fmpos/theses/2014/med/pdf/14M1 69.pdf

9. Koche $T$. Etude des facteurs favorisant la malnutrition chez les enfants de 0 à 59 mois : cas de l'aire de sante de même [internet]. Burkina ; 2011 [cité 28 janv 2016] p. 64. Disponible sur

http://minsantecdnss.cm/sites/minsantecdnss.cm/files/bib lio/fr/2013/etudedesfacteursfavorisantslamalnutritiondese nfantsde 0 à 59 mois. 
10. M, arion, W., Jenkins, et al. Opportunities to improve domestic hygiene practices through new enabling products: A study of hand washing practices and equipment in rural Cambodia. 2013; 23.

Liste des tableaux

Tableau I : Répartition selon les caractéristiques sociodémographiques des mères des enfants malnutris Aigüe Sévère de la Commune VI en 2015.

\begin{tabular}{lcc}
\hline Variables & $\mathbf{N}=\mathbf{3 5}$ & $\%$ \\
\hline Age & & 100 \\
Minimum (16 ans) & 1 & 3 \\
Moyen (24 ans) & 33 & 94 \\
Maximum (43 ans) & 1 & 3 \\
Sexe & & 100 \\
Féminin & 18 & 51 \\
Masculin & 17 & 49 \\
Statut matrimonial & & 100 \\
Mariée & 34 & 97 \\
Célibataire & 1 & 3 \\
Profession & & 100 \\
Ménagère & 21 & 60 \\
Commerçante & 9 & 26 \\
Privé & 4 & 11 \\
Autres* & 1 & 3 \\
Niveau d'instruction & & 100 \\
Non scolarisée & 11 & 32 \\
Primaire & 19 & 55 \\
Non précise & 5 & 13 \\
\hline
\end{tabular}

Autres*:Coiffeuse, Vendeuse, Aide-ménagère.
Tableau II: Répartition des mères selon les conditions d'hygiène et d'assainissements de la famille.

\begin{tabular}{lcc}
\hline $\begin{array}{l}\text { Conditions d'hygiène et } \\
\text { d'assainissements de la famille }\end{array}$ & $\mathbf{n}$ & $\%$ \\
\hline Latrine Moderne & 8 & 23 \\
Latrine Traditionnelle & 27 & 77 \\
& & \\
Pratique du lavage des mains & 26 & 74 \\
Non pratique du lavage des mains & 9 & 26 \\
\hline
\end{tabular}

Tableau III : Répartition des mères selon la période inter génésique

\begin{tabular}{lcc}
\hline Période inter génésique & $\mathbf{n}$ & $\%$ \\
\hline Période intergénégique $<2$ ans & 6 & 17 \\
Période intergénégique $>2$ ans & 22 & 63 \\
Non applicable & 7 & 20 \\
Total & 35 & 100 \\
\hline NB: les Non applicable (NA) étaient des primipares.
\end{tabular}

Tableau IV : Situation du personnel

\begin{tabular}{lccc}
\hline $\begin{array}{l}\text { Categorie } \\
\text { de personnel }\end{array}$ & Formés & Non formés & Total \\
\hline $\begin{array}{l}\text { Médecins } \\
\text { Infirmiers }\end{array}$ & 1 & 0 & 1 \\
\hline
\end{tabular}

